IAU Colloquium 164: Radio Emission from Galactic and Extragalactic Compact Sources

ASP Conference Series, Vol. 144, 1998

J. A. Zensus, G. B. Taylor, \&J. M. Wrobel (eds.)

\title{
Techniques for Analyzing Short Coherence Time VLBI Data
}

\author{
Colin J. Lonsdale \& Sheperd S. Doeleman \\ MIT Haystack Observatory, Westford MA 01886, U.S.A.
}

\begin{abstract}
We have written a suite of programs designed to deal with problems peculiar to short-wavelength VLBI. The most important difference between mm-VLBI and cm-VLBI is that one usually wishes to integrate the data for times which are long compared to the atmospheric coherence time. This causes difficulties with standard VLBI data reduction techniques, and mandates the use of the algorithms implemented in our package for accurate results.
\end{abstract}

Fluctuations in the atmospheric path length, primarily due to water vapor, cause a loss of coherence in interferometric data which becomes severe at millimeter wavelengths. At $3 \mathrm{~mm}$, typical times for a $10 \%$ amplitude loss are in the range of a few seconds. This causes three problems: closure phases are corrupted, sensitivity is reduced, and visibility amplitudes become difficult or impossible to calibrate. To prevent these problems, coherent integration beyond the atmospheric coherence time must be avoided. This in turn leads to very poor sensitivity, the principal limitation of mm-wavelength VLBI.

The Haystack mm-VLBI data reduction package is a subset of the Haystack Observatory Postprocessing System (HOPS). It is based on the theory of incoherent averaging developed by Rogers, Doeleman, \& Moran (1995), and permits the recovery of much of the sensitivity corresponding to fully coherent operation, while preserving the integrity of the amplitudes and closure phases. There are four major steps in the data reduction, which are coordinated and semiautomated by a master shell script called "mmreduce".

The first step is to determine the coherence properties of the dataset. This is done using strong calibrator sources which are detectable within a coherence time. The coherence losses are calculated as a function of coherent integration time, and the resulting curve is fitted to produce an estimate of the coherence time. The fitted coherence times are then decomposed into station-based coherence times, adjusted for elevation, and interpolated to sources which are too weak for direct detection. This produces optimal estimates of the coherence time for each baseline and scan in the dataset.

Next, an incoherent fringe search is performed, which is substantially more sensitive than a coherent fringe search. At $3 \mathrm{~mm}$, typical sensitivity gains are a factor of 2 to 4 for a 6.5 minute scan. The results of the search consist of optimal estimates of rate and delay, but not amplitude or phase.

The third step is visibility generation. The data are incoherently averaged at the fitted values of rate and delay, using a segmentation time which preserves the amplitude information. Segmented triple-products (bispectra) are also formed, which are then averaged separately to produce an optimal and uncorrupted estimate of the closure phase. The reconstructed amplitudes and closure phases are then recombined to create the final visibility data.

The final step involves exporting the visibility data to another software package for further analysis or imaging, as appropriate.

Acknowledgments. Support for the Coordinated mm-VLBI Array work at the Haystack Observatory is provided under a grant from the National Science Foundation to the Northeast Radio Observatory Corporation. 


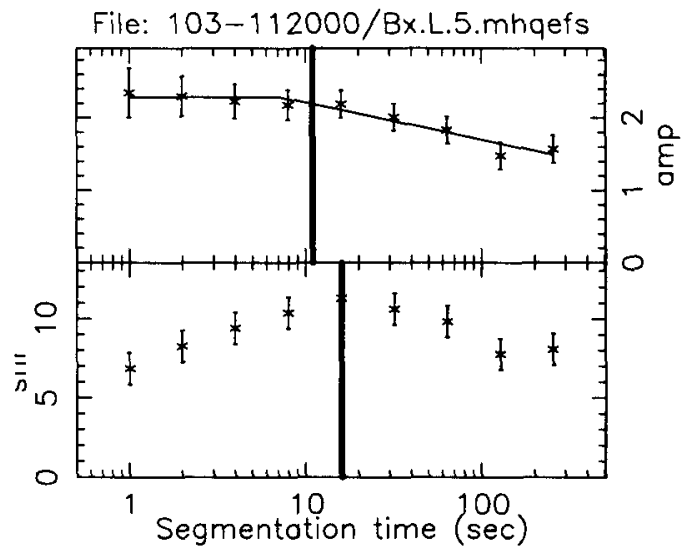

Figure 1. Output of a routine in mmreduce called "cofit" which calculates baseline based coherence times from calibrator scans. Each baseline is fit for two distinct coherence times. The first, a no-loss coherence time, is the segmentation time at which the visibility amplitude falls to $10 \%$ of its maximum (top panel). The lower panel shows the optimal coherence time at which to search for fringes which is typically longer than the no-loss coherence time.

\section{References}

Rogers, A. E. E., Doeleman, S. S., \& Moran, J. M. 1995. AJ, 109, 1391-1401. 